\title{
Effective Mechanism of Graduate Attributes distribution in Engineering Education Curriculum
}

\author{
Seyed M. Buhari ${ }^{1}$, R. Suganya ${ }^{2}$, S. Rajaram ${ }^{3}$ \\ ${ }^{1}$ Department of Information Technology, King Abdulaziz University, Jeddah, Saudi Arabia. \\ ${ }^{2}$ Department of Information Technology, Thiagarajar College of Engineering, Madurai, Tamil Nadu, India. \\ ${ }^{3}$ Head and Registrar (Academics), Department of ECE, Thiagarajar College of Engineering, Madurai, Tamil Nadu, India. \\ ${ }^{1}$ mesbukay@kau.edu.sa \\ ${ }^{2}$ rsuganya@tce.edu \\ rajaram_siva@tce.edu
}

\begin{abstract}
Industries are expecting engineering graduates who possess good attitude along with proficiency in specific domain knowledge to cope with ever-changing working atmosphere. Graduate Attributes (GA), prepared by accreditation authorities, are incorporated into undergraduate programmes to validate whether the graduates from engineering colleges meet such diversified requirements. This study envisioned to evaluate the incorporation of graduate attributes in B.Tech curriculum. As a case study, we have collected B.Tech curriculum of three different universities. All core subjects in the curriculum were distributed across twelve graduate attributes with respective weightage from each subject. We examined the relationships between subjects and corresponding graduate attributes using descriptive statistics. The study revealed that few graduate attributes like GA4 (Conduct Investigation of complex problems), GA6 (Engineer and society), GA7 (Environment and sustainability), and GA11 (Project Management and Finance) have very less weightage $(<40 \%)$ and coverage. Further recommendations are provided to address these concerns through practical sessions and co-curricular activities.
\end{abstract}

Keywords: First Year engineering, Computer programming, logic building, problem solving, debugging, effective academic delivery.

\section{Introduction to Engineering Education Curriculum} Engineering is an activity that is necessary to meet the needs of people, financial development and the stipulation of service to humanity. Engineering education involves the focused application of mathematics, science and a broad knowledge of engineering, technology and methods. Engineers should bring benefits to the society.

The growth of an engineering professional in any of the groups is a continuing process with significant recognized phases. The first phase is the attainment of an accredited educational qualification, the graduate stage. The fundamental purpose of engineering education is to build knowledge, skills and attitudes to enable the graduate to handle any problem with professional ethics and provide solution acceptable to the society. The second phase is professional qualification. The primary purpose of formative growth is to expand competencies to work responsibly as an individual as well as a team member. The third phase is to qualify for the international qualification. The purpose of this phase is to enhance competency throughout their life.

The International Engineering Alliance (IEA) is an umbrella organization that establishes standards for engineering education and competence to practice engineering. The outcome-based education is a requirement to join the Washington Accord (IEA 2013), which was established in 1989. The Washington Accord (WA) is a self-governing, autonomous body that provides mutual recognition of programmes accredited for the engineer track. The graduate attributes approved by the Washington Accord signatories are common to the education of professional engineers in all engineering disciplines. They categorize what graduate should know, the skills they should acquire and the attitudes they should possess. Several countries have accepted the OBA approach in higher education in the early and late 1990s, including the United States (ABET 2011), Australia (Donnelly 2007), and South Africa (Meyer et al, 2010b). The Washington Accord's 12 graduate attributes are GA1 (Engineering knowledge), GA2 (Problem analysis), GA3 (Design and development of solutions), GA4 (Conduct investigation of complex problem, GA5 (Modern tool usage), GA6 (Engineer and society), GA7 (Environment and sustainability), GA8 (Ethics), GA9 (Individual and Team work), GA10 (Communication), GA11 (Project management and finance) and GA12 (Lifelong learning). There are many categories of learning, each of which fall under three major domains - cognitive domain (knowledge level), affective domain (attitude level) and psychomotor domain (skill level). Cognitive domain handles with knowledge and the development of intellectual skills. It comprises GA1 (Engineering knowledge), GA2 (Problem analysis), GA3 (Design/ development of solutions) and GA4 (Investigations). Affective domain includes the manner in which graduates deal with things emotionally (Feelings, appreciations etc.) It comprises GA6 (the Engineer in Society), GA7 (Environment and Sustainability), GA8 (Ethics) and 
GA12 (Lifelong learning). Psychomotor domain involves physical movement, coordination and use of the motor skills (perception and response). It comprises GA5 (Modern tool usage), GA9 (Individual and teamwork), GA10 (Communication), and GA11 (Project / Engineering Management). The purpose of engineering education is to build knowledge, skill and attitude to enable the graduate to proceed to train and experience that will develop the competencies required for independent practice in professional role.

Knowledge, skills and attitudes that engineering graduates ought to possess could be measured using two metrics a) Graduate attributes and b) Course Outcomes. This work presents the distribution of graduate attributes in the Bachelor of Technology (Information Technology) (B.Tech (IT)) programme collected from the three different universities A, B and C. Here in this research article, we have envisioned to evaluate the incorporation of graduate attributes in B.Tech curriculum. All core subjects in the curriculum are distributed across twelve graduate attributes along with the weightage of each subject respectively. Our initial analysis reveals that 4 out of twelve graduate attributes are poorly mapped. We examined the relationships between each subjects and corresponding graduate attributes using the following process: (1) Obtain the curriculum plan with courses mapped to semesters, excluding the elective courses, (2) Map the Course Outcome of each individual core course to graduate attributes, considering the level of mapping, either strong, medium or low, (3) Accumulate the graduate attributes coverage on semester basis considering their coverage levels, (4) Report the program level graduate attributes coverage.

The main contributions of this paper can be summarized as follows:

1. Examined the relationships between each subjects and corresponding graduate attributes.

2. Evaluation of the proposed work on B.Tech curriculum from three different universities A,B and C dataset.

3. Comprehensive experiments were conducted and the importance of twelve graduate attributes distribution across B.Tech programme curriculum was identified.

4. Analyzed the gap between four graduate attributes GA4, GA6, GA7 and GA11 with respect to core subjects in the curriculum.

This paper is organized as follows: literature review is followed by proposed mechanism. Findings from the study based on collected data are presented next. Finally, conclusion along with recommendations is provided.

\section{Literature Survey}

According to the Higher Education Council (HEC) report in Australia, Achieving Quality (HEC,1992), generic attributes are the skills, personal attributes and values which should be acquired by all graduates regardless of their discipline or field of study. In other words, generic skills should represent the central achievements of higher education; as a process such attributes or qualities include critical thinking, intellectual curiosity, problem solving, logical and independent thought, communication and information management skills, creativity and imagination, ethical practice, integrity and tolerance. Similarly, the Dearing report in the UK (National Committee of Enquiry into Higher Education, 1997) states that higher education should 'sustain culture which demands disciplined thinking, encourages curiosity, challenges existing ideas and generates new ones part of the conscience of a democratic society' [Debra et al, 2007]. This emerging importance of generic skills, or graduate attributes in higher education has been influenced by at least the following three factors: the popular perspective that education is a lifelong process; a greater focus on the relationship between education and the employment of graduates; and the development of outcome measures as a part of the quality assurance (Cummings 1998). Research provides insights into how effectively engineering programs can enhance engineering student's Knowledge, Skill and Attitude skills. (Nazzal, 2015) found that Engineering Mathematics subject is difficult among firstyear engineering students. (Suganya, 2019) proposed that collaborative learning of automata theory in Mathematics with domain teacher of Compile Design could help to improve students' knowledge, skill and attitude levels. This outcome suggests that student's confidence in their skills in cognitive level increases over studying a fouryear degree.

The coverage of different graduate attributes by engineering programs remains as a concern for various accreditation authorities. It could be noted that graduate attributes do maintain a balance between technical and nontechnical skills. Students are required to obtain such skills by the time of graduation. Targeting learning outcomes from curriculum has been researched by various researchers (Harden, 2001; Jacobs, 2004; Morehead \& La Beau, 2004; National Council of Teachers of Mathematics, 1989; Plaza, Draugalis, Slack, Skrepnek, \& Sauer, 2007; Uchiyama \& Radin, 2009). It is vital to validate the mappings from curriculum towards learning outcomes to ensure there is matching between what is taught and what is learnt/acquired by students (English, 1984). The process of validating learning outcomes against the curriculum is through course syllabi or course documents developedby course instructors and course coordinators (English, 1984; Harden, 2001; Morehead \& LaBeau, 2004; Willett, 2008). Such a validation activity is required to advance OutcomeBased Education among both instructors and students (Jacobs, 2004, Spencer, Riddle, \& Knewstubb, 2012).

A typical mapping of the course descriptions towards graduate attributes is performed in six stages (Uchiyama \& Radin 2009):

1. Instructors develop mappings for the course learning outcomes to graduate attributes, for their respective courses individually.

2. [Under the umbrella of coordination,] instructors of respective courses meet to review their individual maps. 
3. At the program level [members of a specific committee, quality assurance committee] group themselves as heterogeneous groups to review the mappings for the whole curriculum.

4. Various mappings that require realignment are looked for.

5. Appropriate changes are made to the mappings.

6. Suitable plan is developed and executed.

Thus, mappings of learning outcomes with graduate attribute needs to be verified both at course level (vertical) and program level (horizontal). Such mapping is useful to provide suitable inferences at various levels.

\section{Proposed Mechanism}

Two research questions are considered in this study. Graduate attributes coverage is measured by mapping the course learning outcomes with that of the graduate attributes. The presence of many courses catering a certain program outcome indicates that the students are well abrupted with the necessary skill. In contrast, poor coverage of a program outcome indicates that students might not have the necessary skill for a successful career.

A. Research Question 1 (RQ1): To what extent are the proposed graduate attributes covered in the core courses of the engineering program?

This study proposes to infer about the pattern of coverage of any specific program outcome from the third to the eighth semester of the engineering program. Certain knowledge-based graduate attributes are expected to be covered during the early years of study. Moreover, graduate attributes that address applications or creativity, which are in the higher level of Bloom's taxonomy is expected to be covered more during the later years of study.

B. Research Question 2 (RQ2): What is the proportion of coverage of the graduate attributes over different semesters of the engineering program?

Coverage of graduate attributes are studied from the perspective of different semesters to know what a student accomplishes and when. This could also help the program in-charge to plan for internship and other student exchange programs.

\section{Data Sources}

Three four-year full-time engineering programs from different universities were considered in this study. All the three programs considered are accredited by National Board of Accreditation (NBA) in India. These programs cover the area of Computing and Electronics. A total of 143 courses were studied in this research. Out of these 143 courses, mapping to GAs were not available for 49 sources. Thus, the coverage of courses was curtailed to 94 courses. In order to analyze the programs, all the core or compulsory courses were reported in a google sheet. Individual courses were indicated through their course learning outcomes along with their respective mappings towards graduate attributes. The choice of core courses only is to ensure observations or inferences made are applicable to all graduates and not certain specific percentage of graduates.
Detailed descriptions were available for each of the program. These include the following: (1) weekly coverage, (2) hours allocated for each course contents, (3) course learning outcomes, (4) weightage of course learning outcomes, (5) mapping of course learning outcomes to graduate attributes, (6) objectives of the course, (7) textbook followed, (8) assessment policy, (9) prerequisite, (10) semester of study and others. The mapping of the course learning outcomes to graduate attributes is of three levels: low, medium and strong.

\section{Data Analysis}

Course outlines from these three engineering programs were documented into a google sheet. The respective mappings are updated on individual sheets. After the individual course mappings and relevant information is obtained, program level validation is performed. Here, the courses are also segregated based on their semester of study. The analysis for this research involves the following stages:

1. The whole curriculum plan, considering only the core courses, is accumulated to a single table covering various relevant information like course code, course name, number of credits, semester of study, prerequisite, suitable coverage of graduate attributes. Bloom's taxonomy coverage is also reported, if available.

2. Semester-wise coverage of each GA is reviewed.

3. Along with semester-wise coverage of GA, the levels of each GA are also studied.

4. Furthermore, Bloom's taxonomy level coverage over different semesters is also analyzed.

The study reveals how various GAs are covered in the curriculum. Visual representation could provide some insight into how the GAs are addressed by the curriculum. Furthermore, the mappings of different GAs from similar courses at different universities could also be studied.

\section{Results and Discussion}

The research question 1 (RQ1) "To what extent are the proposed graduate attributes/graduate attributes covered in the core courses of the engineering program?" is answered through the following analysis. In the engineering program, we have considered courses over various semesters: 9 courses from semesters 2, 8 from semesters 3, 9 from semesters 4,8 from semesters 5,5 from semesters 6 and 5 from semesters 7. The distribution of Graduate attributes over these semesters are shown in Figure 1. It could be observed from figure 1 that certain graduate attributes like GA4 [Conduct Investigation of complex problems], GA6 [The engineer and society], GA7 [Environment and sustainability] and GA11 [Project management and finance] are poorly covered. Among these poorly covered GAs, GA6 and GA7 are non-technical skills while GA4 and GA11 are technical skills. Later graduate attributes, like GA8 to GA12, are better covered in later semesters. As an observation, GA7 coverage for semester 4 is poorly covered. Similarly, GA4 is not covered suitably in semester 3. The poorly covered GA4 and GA11 are better addressed in semester 4 . 


\begin{tabular}{|c|c|c|c|c|c|c|c|c|c|c|c|c|c|}
\hline Semesters & GA1 & GA2 & GA3 & GA4 & GA5 & GA6 & GA7 & GA8 & GA9 & GA10 & GA11 & GA12 & $\begin{array}{l}\text { No. of } \\
\text { Courses }\end{array}$ \\
\hline Semester 2 & 49 & 41 & 31 & 6 & 27 & 7 & 16 & 21 & 26 & 25 & 3 & 32 & 9 \\
\hline Semester 3 & 44 & 46 & 42 & 2 & 32 & 9 & 9 & 33 & 18 & 28 & 7 & 45 & 8 \\
\hline Semester 4 & 48 & 45 & 29 & 21 & 23 & 9 & 1 & 17 & 24 & 27 & 14 & 27 & 9 \\
\hline Semester 5 & 39 & 39 & 33 & 7 & 26 & 6 & 7 & 23 & 23 & 15 & 10 & 27 & 8 \\
\hline Semester 6 & 25 & 25 & 18 & 5 & 15 & 5 & 6 & 16 & 18 & 16 & 1 & 13 & 5 \\
\hline Semester 7 & 27 & 27 & 19 & 6 & 15 & 9 & 4 & 18 & 17 & 19 & 10 & 18 & 5 \\
\hline TOTAL & 232 & 223 & 172 & 47 & 138 & 45 & 43 & 128 & 126 & 130 & 45 & 162 & 44 \\
\hline$\%$ Coverage & 15.11 & 14.53 & 11.21 & 3.06 & 8.99 & 2.93 & 2.8 & 8.34 & 8.21 & 8.47 & 2.93 & 10.55 & \\
\hline
\end{tabular}

Table 1. Semester wise GA coverage

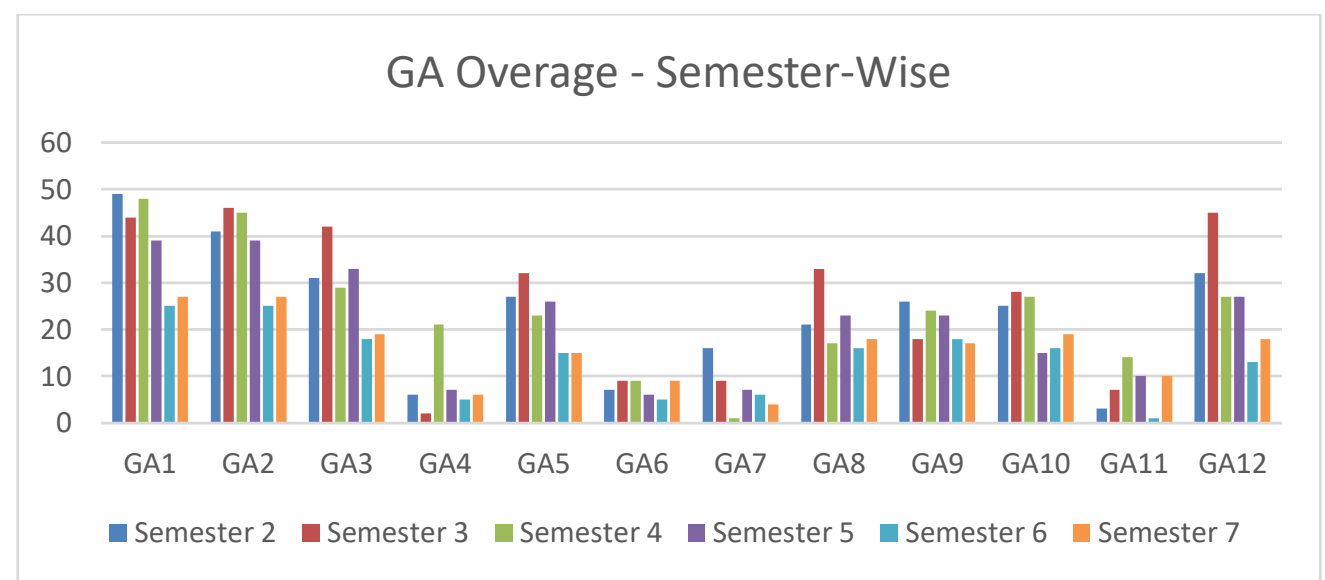

Figure 1. Semester wise GA coverage

The research question 2 (RQ2) "What is the proportion of coverage of the graduate attributes over different semesters of the engineering program?" is answered through Table 1. GA1, GA2, GA3 and GA12 are amicably addressed over different semesters of the engineering program. Meanwhile, GA5, GA8, GA9 and GA10 are reasonably addressed over different semesters. Exclusion of semester 4 in GA4 and semester 2 in GA7 will indicate very poor coverage of these two GAs.

Further study on three levels of different GAs are elaborated in figures 2 and 4 . GAs 1 to 4,5 to 8 , and 9 to 12 are shown in figures 2, 3 and 4 respectively. Strong levels of GAs 1, 5, 8, 9 and 10 are covered better than the other levels. Meanwhile, GAs 2, 6, 7, and 12 are covered more in medium levels. It should be noted that Low levels of GAs 2 , $3,4,5,8$, and 9 are covered over different semesters. Comparing individual GAs over different semesters indicates a trend of coverage as normally distributed with higher coverage during semesters 3, 4 and 5. Also, poorly covered GAs has no coverage of strong levels in various semesters. This is one of the important concerns to be addressed. 


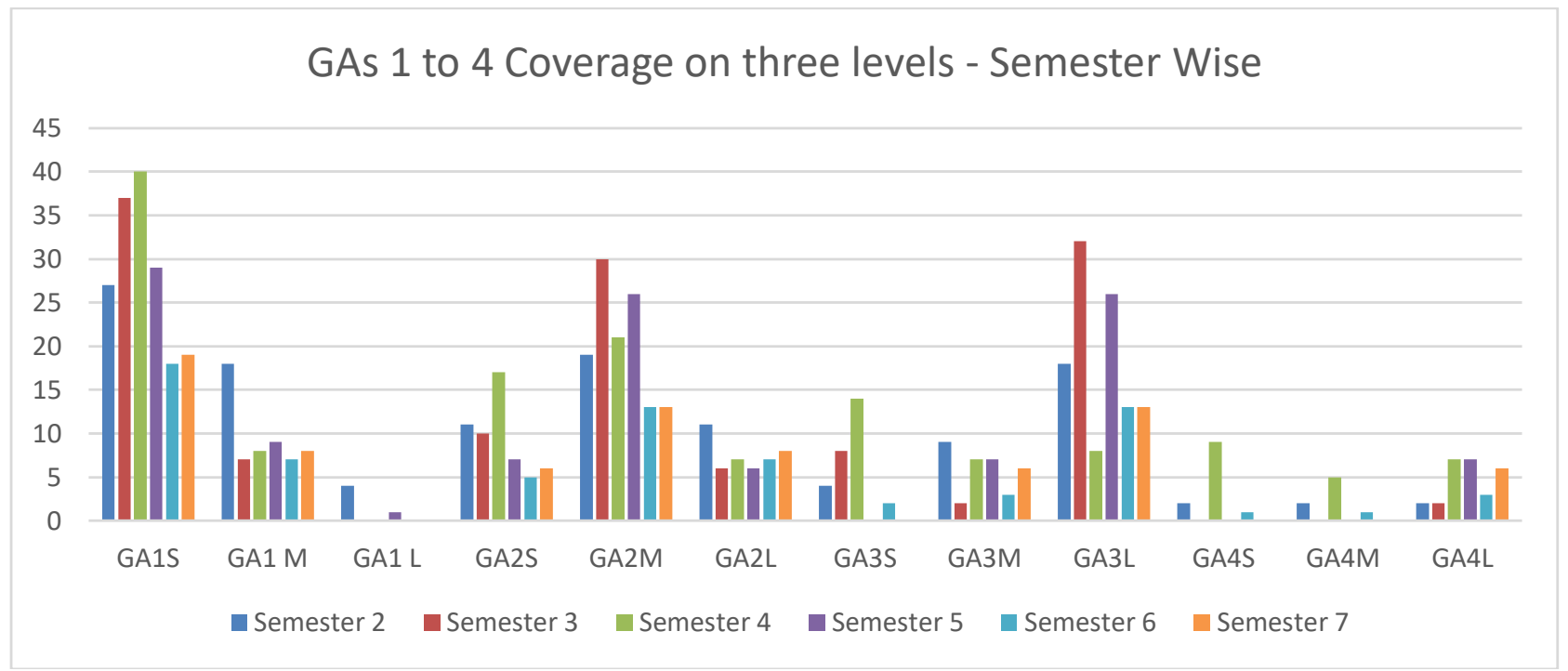

Figure 2. Semester wise GAs 1 to 4 with three levels coverage

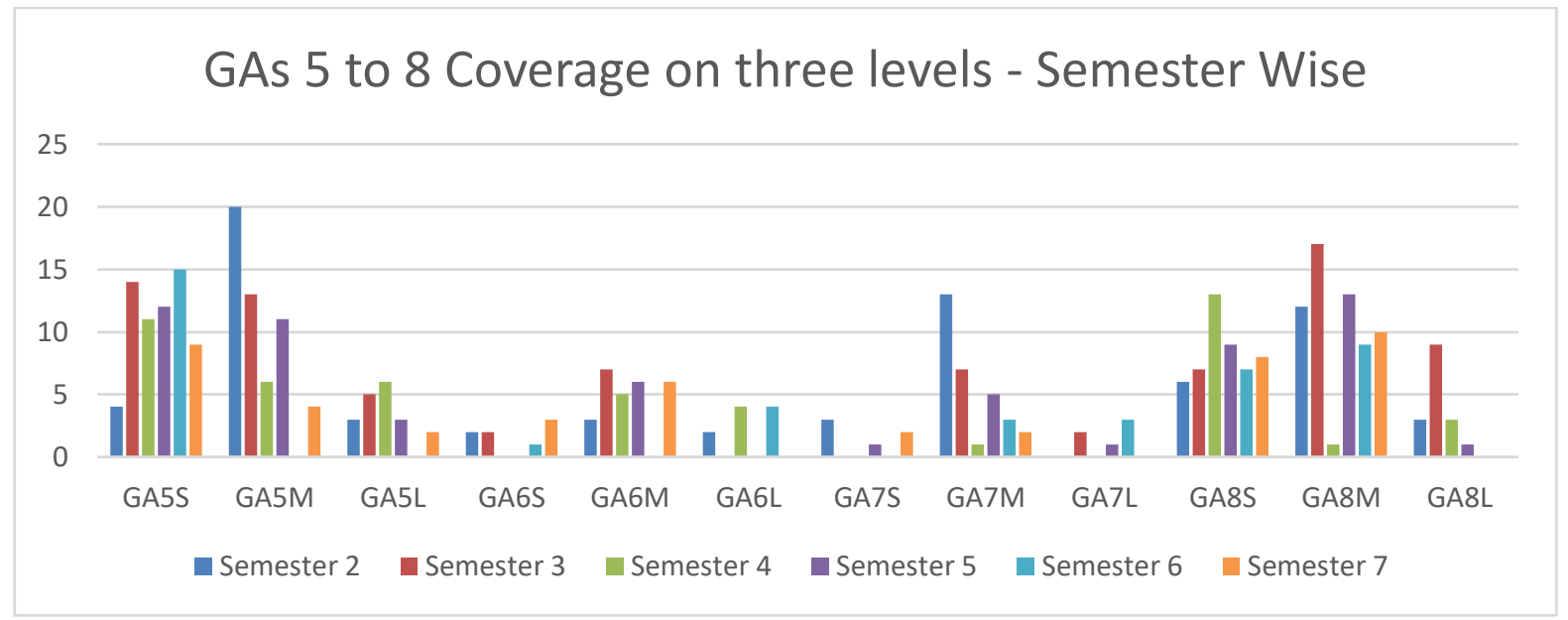

Figure 3. Semester wise GAs 5 to 8 with three levels coverage

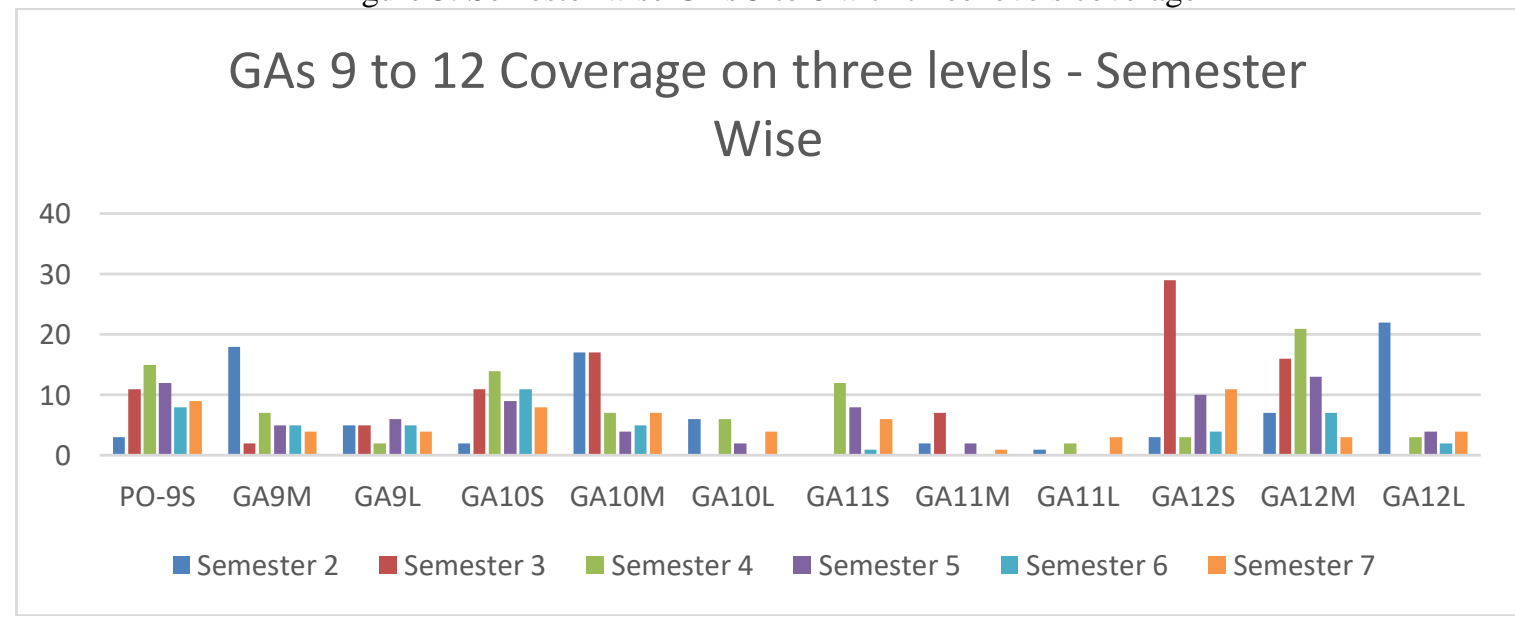

Figure 4. Semester wise GAs 9 to 12 with three levels coverage

Extending the study from GAs to Bloom's taxonomy coverage over semesters was also performed. The outcome of such a study is shown in Figure 5. Bloom's taxonomy level "Applying" is the most addressed in the curriculum. This confirms that the proposed engineering curriculum emphasizes on applying concepts onto different domains. Early semesters have higher coverage of understanding and remembering. One major concern here in the Bloom's taxonomy coverage is the poor representation of evaluating and creating domains. These two are necessary towards development of "innovation" skills among graduates. 


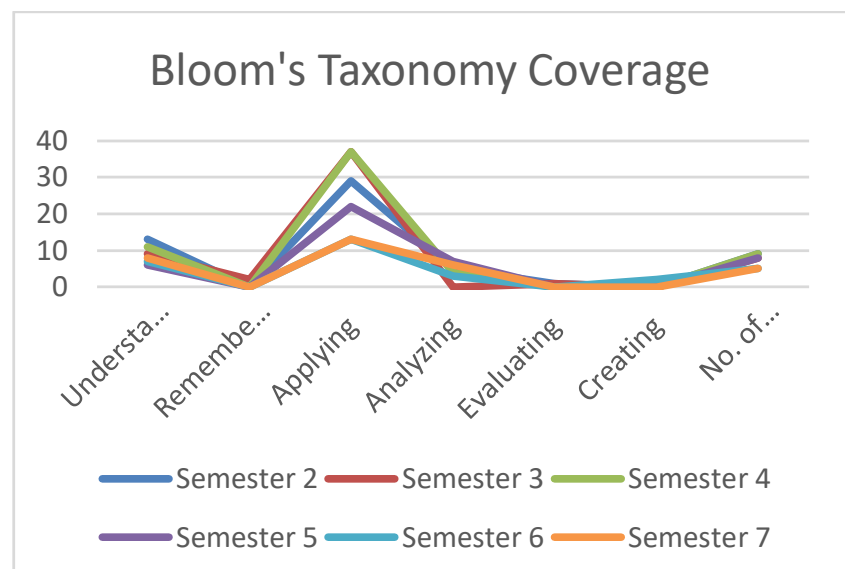

Figure 5. Semester wise Bloom's Taxonomy coverage

In summary, certain GAs and Bloom's taxonomy are poorly covered in the engineering curriculum. Coverage of GA2 [Problem Analysis] could be enhanced further to cater for GA11 [Project management and finance]. Similarly, the curriculum needs to be enriched with necessary nontechnical skills. May be, the coverage of internship and capstone project could help evaluate non-technical skills attainments among engineering graduates. Certain "Applying" covered aspects could be transferred towards evaluating and creating domains.

\section{Conclusion}

This research study provides insights into proper planning of the program and their respective courses. This study improves our understanding on the underlying content courses and knowledge base of courses recommends academic institutions to provide appropriate structural procedures while defining and enhancing a curriculum. The study also discovered the solution for expanding the coverage of these four graduate attributes through CDIO Curriculum. GA4 can be addressed through the elective and industry supported credit courses with hands-on practices for solving real time problems. Both GA6 \& GA7 can be attained by involving students in more co-curricular activities like NSS, NCC etc. to gain exposure from problems in the societal arena. Students can be motivated to take part in 'Engineering Exploration', 'Lateral thinking' and 'Design Thinking' courses in subsequent semesters and make participation in Hackathon and Industries conducting crowd sourcing contest to solve real time problems for sustainable development by using professional engineering practices. GA11, closely related to projects, can be developed by making students to engage in summer internship program, mini- projects and semester projects. Also, we can encourage student to conduct Inter-college programs and societal events to explore project management activities in multidisciplinary environments. Considering only three programs could be one of the limitations of this work.

\section{Future work}

As a future work, closely related engineering disciplines like that of computer science and engineering with Information Technology could be studied for their course objectives to GA mappings. Division of GAs into technical and non-technical and identifying courses that cater for these types of GAs could reveal certain concentrations from different streams of courses. Effective use of assessments to measure the attainment levels of different GAs should also be studied further. Focus on specific GAs by certain courses starting from low level to strong level of coverage also could be used to identify the interests of students and their respective choice of tracks or specialization. Certain interests among faculty members could also be inferred by referring to the GA mappings along with the Bloom's taxonomy selection. Thus, assisting the administration towards identifying potential faculty development programs.

\section{References}

1. Accreditation Board for Engineering and Technology (ABET). 2011. Criteria for accrediting engineering programs. ABET, Baltimore, Maryland

2. Cummings, R. (1998, February). How should we assess and report student generic attribute? Paper presented at the 7th Annual Teaching and Learning Forum, Nedlands, Australia.

3. Debra Bath, Calvin Smith, Sarah Stein, \& Richard Swann, (2007). Beyond mapping and embedding graduate attributes: bringing together quality assurance and action learning to create a validated and living curriculum

4. Donnelly, K. 2007. Australia's adoption of outcomes based education: A critique. Issues in Educational Research, 17: 183-206.

5. English, F. (1984). Curriculum mapping and management. In B. D. States (Ed.), Promoting school excellence through the application of effective schools research: Summary and proceedings of a 1984 regional exchange workshop, Nashville, Tennessee, April 15-16, 1984. Charleston, WV: Appalachia Educational Laboratory.

6. Harden, R. (2001). AMEE Guide No. 21: Curriculum mapping: A tool for transparent and authentic teaching and learning. Medical Teacher, 23(2), 123-137.

7. International Engineering Alliance (IEA). 2013. Washington Accord. Available from http://www.washingtonaccord.org/WashingtonAccord [Accessed on2 March 2013].

8. Plaza, C. M., Draugalis, J. R., Slack, M. K., Skrepnek, G. H., \& Sauer, K. A. (2007). Curriculum Mapping in Program Assessment and Evaluation. American Journal of Pharmaceutical Education, 71(2), 1-8. http://dx.doi.org/10.5688/aj710220

9. Jacobs, H. H. (2004). Getting results with curriculum mapping. Alexandria, VA: Association for Supervision.

10. L. J. Nazzal, (2015) "Engineering creativity: Differences in creative problem solving stages across domains," Ph.D. dissertations, Graduate School, Univ. Connecticut, Mansfield, CT, USA, 2015. 
11. Higher Education Council (1992). Achieving quality. Canberra: Australian Government Printing Service.

12. Meyer, L., Lombard, K., and Wolhuter, C. 2010 b. Outcomes-based assessment for South African teachers. Van Schaik Publishers, Hatfield, Pretoria, South Africa.

13. Morehead, P., \& LaBeau, B. (2004). Successful curriculum mapping: Fostering smooth technology integration. Learning and Leading with Technology, 32(4), 12-17.

14. National Council of Teachers of Mathematics. (1989). The curriculum and evaluation standards for school mathematics. Reston, VA: NCTM.

15. Spencer, D., Riddle, M., \& Knewstubb, B. (2012). Curriculum mapping to embed graduate capabilities. Higher Education Research \& Development, Ê31 (2) Ê217-231. http://dx.doi.org/10.1080/07294360.2011.554387

16. Suganya Ramamoorthy, M. Kameswari, (2019). Transformation of cognitive level in interfacing automata theory and Grammar with Compiler design and its applications through collaborative teaching and learning, presented in IUCEE on ICTIEE 2019 at Chitkara University, Punjab, Jan 10-11, 2019.

17. Uchiyama, K. P., \& Radin, J. L. (2009). Curriculum mapping in higher education: A vehicle for collaboration. Innovations in Higher Education, 33(4), 271-280. http://dx.doi.org/10.1007/s10755-008-9078-8

18. Willett, T. G. (2008). Current status of curriculum mapping in Canada and the UK. Medical Education, 42(8), 786-793. http://dx.doi.org/10.1111/j.13652923.2008.03093.x PMid:18564300 